\title{
AN HYBRID MODEL FOR EVALUATING THE OVERALL PERFORMANCE OF GYNECOBSTETRICS DEPARTMENT: AN APPROACH BASED ON FAHP, DEMATEL AND TOPSIS
}

\begin{abstract}
Hospitals typically lack effective service level strategic and operational planning. Some of them do not have a good "quality" organization. In most of the cases, they could use some optimization models to try to improve it. The point of seeking care at a hospital is to get well. That may seem obvious, but it is a fact that sometimes gets lost in discussions over the availability of "quality" health care. The aim of the present research is to investigate the relationship between the organization and performance of health service systems. In detail, the gynecobstetrics department. The study assesses several variables such as the quality of medical equipment, the quality of care, the patient safety etc. A multi criteria approach based on Fuzzy Analytic Hierarchy Process (FAHP), Decision Making Trial and Evaluation Laboratory (DEMATEL) and the Technique for Order of Preference by Similarity to Ideal Solution (TOPSIS) is proposed. The research concerns three hospitals from the public sector of Colombia. The results show the most critical variables and the correlation among all selected criteria of performance.
\end{abstract}

Keywords: FAHP, DEMATEL, TOPSIS, gynecobstetrics department, performance.

\section{Introduction}

In 1993, the health care system in Colombia was publicized as a paradigm to be copied across the developing world (del Pilar et al., 2017). The Colombian mandatory system of quality assurance (SOGCS, Sistema Obligatorio de Garantía de Calidad en Salud) includes a series of methodological elements to assess the quality of the health services. Colombia has significantly improved its health system over the past 20 years, leading to a rise in life expectancy and a fall in infant mortality. Infant mortality has fallen from 40 deaths per 1000 live births in 1970 to 12.8 and life expectancy at birth reached 75.2 years in 2013. To maintain its ambition of universal, high-quality health care, Colombia should now focus on improving efficiency and strengthening financial sustainability, according to a new OECD report (OECD, 2016).

All Colombians have access to the same health care services and have good financial protection from excessive health care spending if they get sick. Out-of-pocket expenditure paid by patients is only $14 \%$ of total health spending. This is one of the lowest proportions in Latin America and is lower than the OECD average of $19 \%$. Unmet health care needs and waiting times for medical appointments have been significantly reduced.

However, the health data infrastructure in Colombia still needs improvement, but efforts are now in place to standardise patient outcomes data. Disease registries for select conditions have been in place since 2007, when the Ministry of Health created an independent organisation, Cuenta de Alto Costo, in order to reduce accessrelated inequalities for patients with cost-intensive diseases.

To this regard, to determine a hospital's quality of care, it is important to look at different aspects of care. It is important to consider many different measures to create a more holistic picture of the quality of health care each International Symposium on the Hong Kong, HK. Analytic Hierarchy Process July 13 - July 15, 2018 
hospital delivers. In Colombia, an ambitious reform programme includes among its measures the attempt to universalize a segmented health system and the transformation of public hospitals into "autonomous state entities". By design, the Colombian reform programme avoids the forces that produce equity losses in other developing countries (McPake et al., 2003).

The format of this paper is as follows. In Section 2 a brief review of the literature is presented. In Section 3 the hypotheses and objectives are introduced and research design is then developed in Section 4 . A numerical investigation has been provided in Section 5. A summary of main limitations and main contributions of this paper are provided in Section 6 and Section 7.

\section{Literature Review}

Hospitals are critical elements of the health care system. In recent years the demand for their services has increased greatly and in response they have become larger and more sophisticated.

The power of hospital performance management today is recognized for providing effective healthcare quality. One of the basic objectives pursued by most countries is to improve their health system both in terms of quality services and efficiency and the extent to which its resources are put to good use (Ortíz-Barrios et al., 2017).

There are many ways to measure the performance in health care system. A multi criteria approach based on the integration of Multiple-criteria decision-making (MCDM) is believed to be the best way to perform a overall analysis of hospital quality service. Our review of the literature indicates that approaches for identifying hospital quality service from a multi-criteria viewpoint are limited.

MCDM methods represent the way how to make choices in case that multiple criteria are present. In many reallife problems more than one criterion is observed and evaluation of such problems requires application of MCDM approaches (Váchová and Hajdíková, 2017). There are different categories of MCDM methods. In the present research, three methods have been proposed, as explained below:

- The Fuzzy analytic hierarchy process (AHP) proves to be a very useful methodology for multiple criteria decision-making in fuzzy environments, which has found substantial applications in recent years. The vast majority of the applications use a crisp point estimate method such as the extent analysis or the fuzzy preference programming (FPP) based nonlinear method for fuzzy AHP priority derivation.

- The Decision-Making Trial and Evaluation Laboratory (DEMATEL) methodology is proposed to for researching and solving complex and intertwined problem groups because of its capability in verifying interdependence between variables and try to improve them by offering a specific chart to reflect interrelationships between variables.

- The Technique for Order Preference by familiarity to Ideal Solution (TOPSIS) is a method that ranks the alternatives based on their distance from ideal and basal alternatives.

Based on the above mentioned methods we were able to identify the best performing hospitals, as well as the worst performing ones. The deeper analysis is definitely needed in order to understand what the cause of their positions in the ordered list of hospitals actually is..

\section{Hypotheses/Objectives}

The problem herein described reflects the concern relating to the performance of gynecobstetrics departments and its influence on risk of fetal, infant and maternal mortality rates. These units should be then provide wide accessibility and efficiency whilst delivering high quality and on-time medical care.

Our research is based on the awareness that each year, families in low and middle income (LMIC) countries face the death of approximately 4 million new- borns, 3 million fetuses and 300,000 pregnant women (99\% of those that occur worldwide) 
From this point of view it is worth to note that Colombia is in the midst of a demographic transition resulting from steady declines in its fertility, mortality, and population growth rates. A population pyramid illustrates the age and sex structure of a country's population and may provide insights about political and social stability, as well as economic development. The population is distributed along the horizontal axis, with males shown on the left and females on the right. The male and female populations are broken down into 5-year age groups represented as horizontal bars along the vertical axis, with the youngest age groups at the bottom and the oldest at the top. The shape of the population pyramid gradually evolves over time based on fertility, mortality, and international migration trends

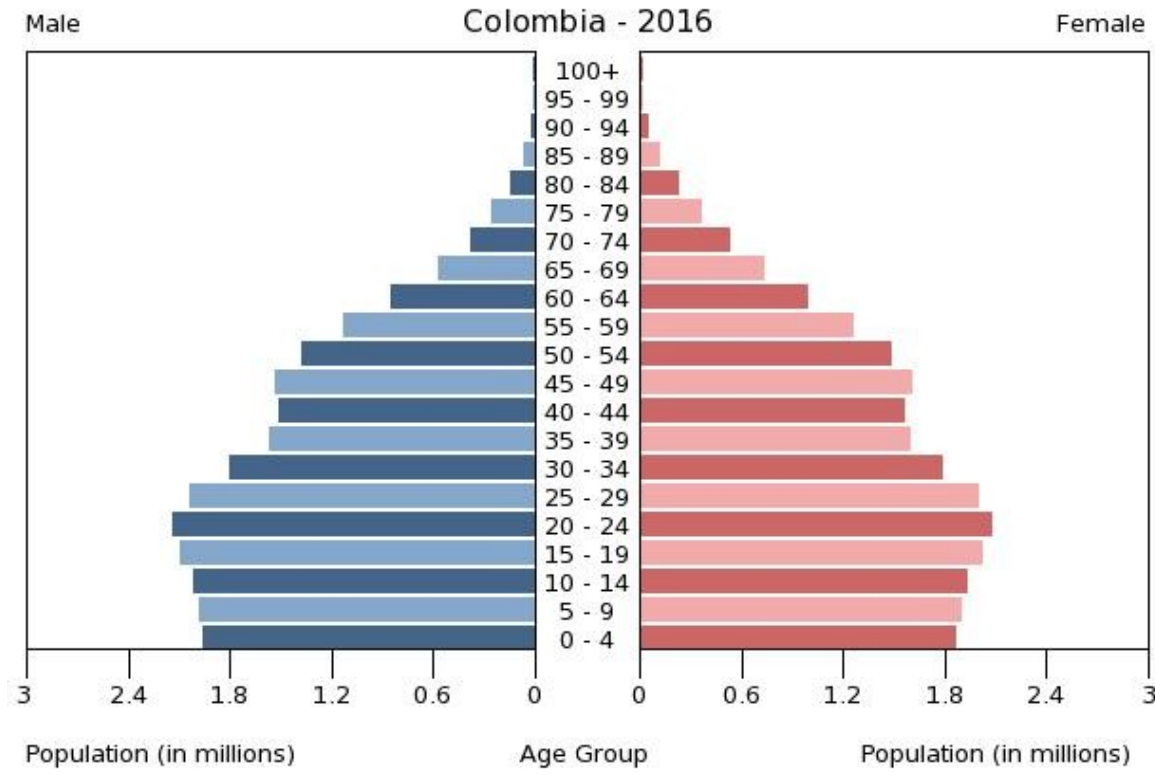

Figure 1: Colombian population pyramid (2016)

Considering the aforementioned aspects, this research aims to evaluate the overall performance of gynecobstetrics departments in a hospital public sector of Colombia.

\section{Research Design/Methodology}

The present study is based on the integration of the Fuzzy Analytic Hierarchy Process (FAHP), Decision Making Trial and Evaluation Laboratory (DEMATEL) and the Technique for Order of Preference by Similarity to Ideal Solution (TOPSIS). FAHP is used to calculate criteria and sub-criteria weights under uncertainty. Then, DEMATEL is applied to determine the interdependence and feedback between decision elements and TOPSIS ranks the hospitals with basis on the concept that the chosen alternative should have the shortest geometric distance from the positive ideal solution and the longest geometric distance from the negative ideal solution.

\section{Data/Model Analysis}

A decision-making model with 8 criteria, 30 sub-criteria and 3 alternatives (hospitals) has been designed. The model has been developed with the support of several experts from the healthcare sector and studies developed by the Ministry of Health and Social Protection. Figure 2 presents the proposed MCDM model. 


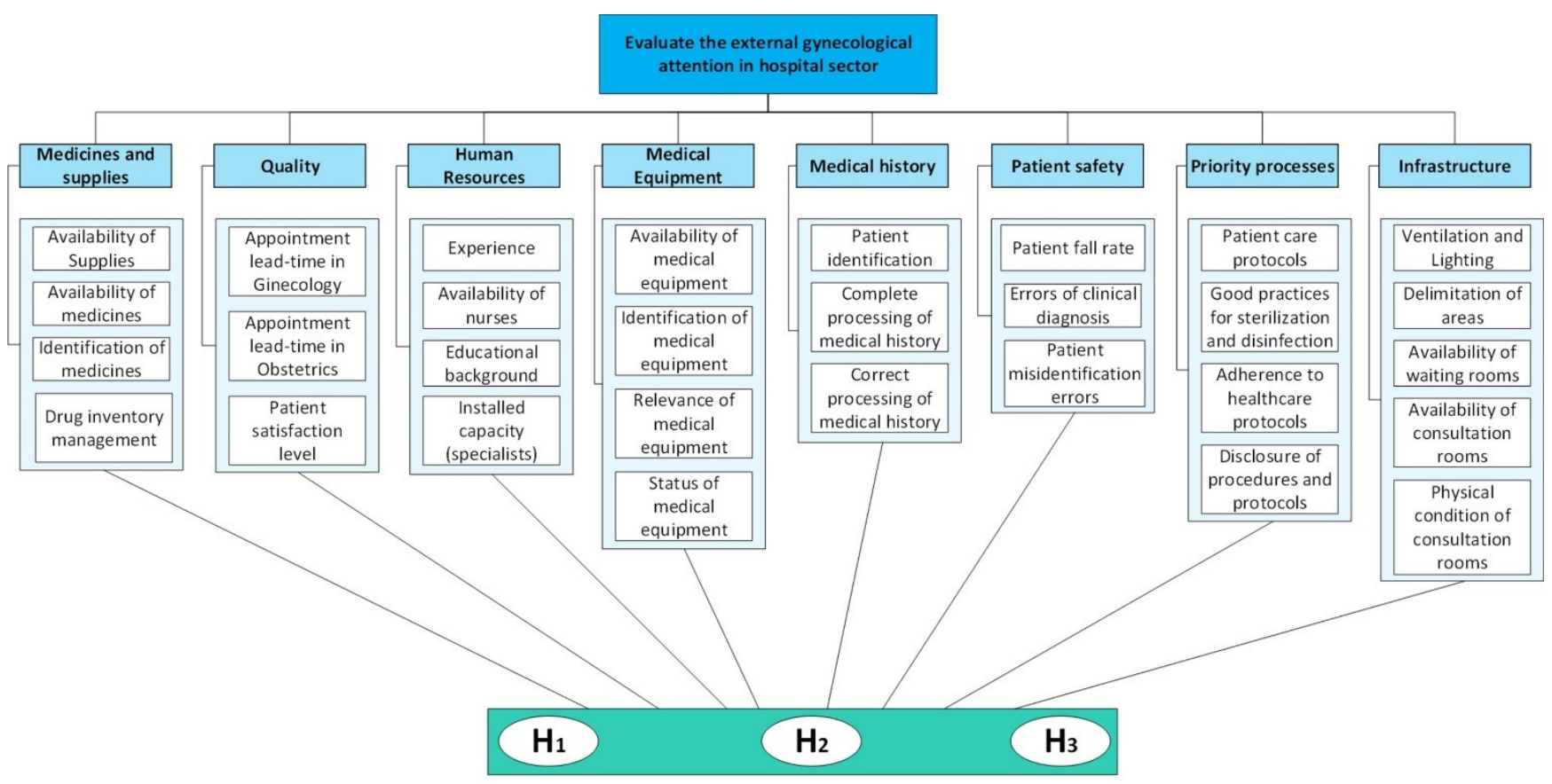

Figure 2: The proposed decision-making model for evaluating the external gynecological attention in hospital public sector

The results indicate that the most significant criteria when evaluating the overall performance of gynecobstetrics departments of hospital public sector are: PATIENT SAFETY (GW $=15.59 \%)$, QUALITY $(\mathrm{GW}=15.27 \%)$ and PRIORITY PROCESSES (GW = 13.06\%). However, there is not a significant gap between the most and least significant criteria (4.81\%) and improvement strategies must be therefore multi-criteria to be highly effective. The most important sub-criterion was PATIENT MISIDENTIFICATION ERRORS (6.17\%) which can be explained by the increasing interest on implementing decision support systems and patient safety programs aiming to tackle this problem. On the other hand, PATIENT SAFETY, QUALITY and PRIORITY PROCESSES were found to be the most influencing factors and should be then prioritized for continuous improvement. Finally, after implementing this approach in a hospital public sector comprised of three hospitals (refer to Fig. 2), it was concluded that $\mathrm{H} 1$ is the best hospital with a closeness coefficient of 0.8031 . In addition, potential improvement opportunities were identified for each hospital in order to augment the cluster competitiveness.

\section{Limitations}

It is important to note that the findings may be related to the characteristics of the analyzed scenario. The research was limited to three hospitals from the public sector of Colombia which could partially explain the results. Future studies will take into account interactions between this department and others that may affect its operability.

\section{Conclusions}

Many countries are experimenting with public hospital reform in order to improve the quality of their services. This study attempts to propose a strategy to assess the quality assessment of the health service in the Colombian health system. In particular, as a case study, the quality of the gynecobstetrics department is analyzed. The study uses three complementary techniques to address the problem. Results show that it is a powerful approach 
understanding the problem and gaining valuable insight into the inner workings of the Colombian Health System.

\section{Key References}

del Pilar Villamil, M., Barrera, D., Velasco, N., Bernal, O., Fajardo, E., Urango, C., \& Buitrago, S. (2017). Strategies for the quality assessment of the health care service providers in the treatment of Gastric Cancer in Colombia. BMC Health Serv Res. 17: 654.

McPake, B., Yepes, F.J., Lake, S., \& Sanchez, L.H. (2003). Is the Colombian health system reform improving the performance of public hospitals in Bogotá? Health Policy Planning, 18(2), 182-94.

Ortíz-Barrios, M.A., Escorcia-Caballero, J.P., Sánchez-Sánchez, F., De Felice, F., Petrillo, A. (2017). Efficiency Analysis of Integrated Public Hospital Networks in Outpatient Internal Medicine. Journal of Medical Systems 41(10), 163 .

OECD Reviews of Health Systems: Colombia 2016.

Váchová, L., \& Hajdíková, T. (2017). Performance of Czech hospitals: comparison with ideal solution, in Proceedings of EFS 2017 - European Financial Systems. 